\title{
Optimization of Cosine Modulated Filter Bank for Narrowband RFI
}

\author{
Yingsi Liang \\ Department of Electrical Engineering \\ Southern Methodist University \\ Dallas, Texas 75275 \\ Email: yliang@smu.edu
}

\author{
Oren E. Eliezer, Member, IEEE \\ Xtendwave Inc. \\ Dallas, Texas 75254 \\ Email: oeliezer@xtendwave.com
}

\author{
Dinesh Rajan, Senior Member, IEEE \\ Department of Electrical Engineering \\ Southern Methodist University \\ Dallas, Texas 75275 \\ Email: rajand@lyle.smu.edu
}

\begin{abstract}
An optimization scheme is developed for a cosine modulated perfect- reconstruction (PR) filter bank that is tailored to mitigate the effects of narrowband radio frequency interference (RFI). The conventionally used optimization criterion for bandpass filtering is to maximize the sidelobe attenuation by minimizing the stopband energy and minimizing the maximum stopband ripple. The proposed optimization scheme is designed particularly to combat RFI with completely known or partially known statistics. Simulation results for the scenario of strong narrowband RFI show that the proposed optimization scheme offers about $14 \%$ improvement in the system's data throughput when compared to the traditional schemes.
\end{abstract}

\section{INTRODUCTION}

Multi-carrier modulation (MCM) techniques are widely used in Digital Subscriber Loop (DSL) communications because of their capability to combat frequency-selective channels. The most commonly used modulation scheme is Discrete Multi-tone (DMT), which uses rectangular truncated sinusoids of mutually orthogonal frequencies as sub-carrier waveforms. Although it benefits from an efficient implementation using DFT, its sub-carriers exhibit poor spectral containment. The overlap between sub-carriers results in performance degradation, especially in the presence of RF interference (RFI).

RF sources which share the same spectra with DSL could cause strong interference and significantly degrade the system performance. According to [1], as many as $30 \%$ of residential DSL systems in the US experience RFI at the levels of - $30 \mathrm{dBm}$ or above. Several schemes have been proposed to address such interference problems. A commonly adopted solution is to filter the sub-carriers with raised cosine windows, thus further limiting their spectral containment and allowing them to be more robust to narrowband RFI. However, the waveforms after windowing still have relatively high energy in their first sidelobe [2]. Wavelet packet based multi-carrier modulation (WP-MCM) is proposed as an alternative modulation scheme with better spectral containment [3], [4]. However, the tree structure filter bank results in mirror image spectral properties, which makes it difficult to implement the frequency-division duplex (FDD) required for a standard DSL application [5]. Discrete Wavelet Multi-tone (DWMT) is fundamentally the same as WP-MCM because they both enable the symbol to extend beyond the transform size while still maintaining orthogonality. However, DWMT transceivers use a cosine modulated filter bank (CMFB), which is a parallel structure filter bank, so that all the sub-carriers have the same bandwidth and evenly shifted central frequency. Further, DWMT requires a simple equalizer with only two taps per sub-carrier [6] and is thus a more practical scheme than WP-MCM.

The prototype filter design is crucial in DWMT because it determines the frequency selectivity of the sub-carriers. A common approach to optimize the filter design is to maximize the stopband attenuation, leading to two filter design criteria: least square (LS) and peak-constrained or minimax method [7]-[10]. The former tries to minimize overall stopband energy and the latter aims at minimizing the maximum stopband ripple. Optimization involving minimizing inter-symbol interference (ISI) and inter-carrier interference (ICI) has also been studied [11]. Although suppressed stopband frequency response can lead to improved robustness against RFI, its optimality in minimizing the effect of RFI is not proven.

In this paper, we propose an optimal RF interference mitigation scheme for DWMT. The optimization is performed in two cases: i) The RFI is caused by static and predictable sources such as AM broadcast radio, and ii) The RFI is caused by unpredictable sources such as amateur (ham) radio. In the former case, a training sequence can be sent periodically to obtain knowledge of the RFI signal such as its center frequency. In the latter case, or if increased system complexity is not desirable, a training sequence is not implemented. In this case, we assume the center frequency of the RFI is confined in a certain known frequency range, with bandwidth that is narrower than the system bandwidth. The prototype filter optimization is based on a lattice filter structure, and thus the CMFB can be calculated with low computational complexity [9]. A performance comparison of the proposed optimization scheme with other commonly used optimization schemes, as well as WP-MCM and DMT systems, is also provided and demonstrates the superiority of the proposed optimization scheme. The relationship between the system performance and the center frequency of the RFI is also studied.

Section II presents an introduction to DWMT and to the commonly adopted optimization approach. In Section III, the proposed optimization scheme is discussed, for which simulation results are given in Section IV. 


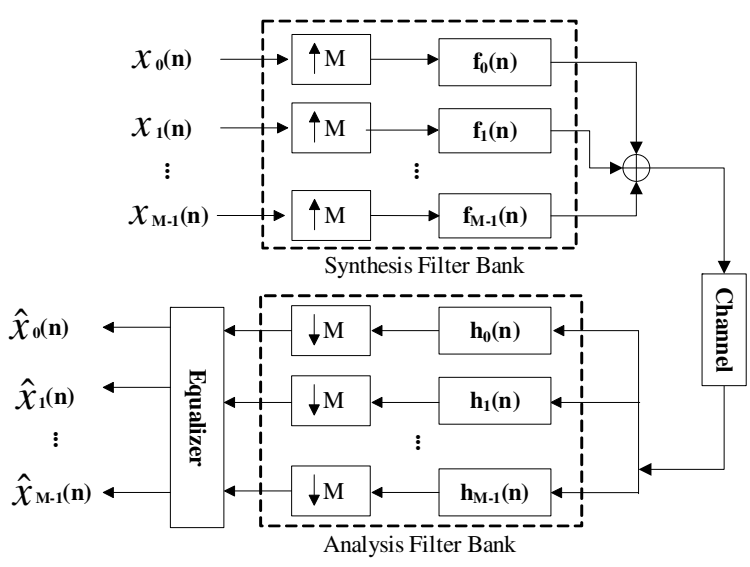

Fig. 1. M-subband filter bank of DWMT

\section{Discrete WAVElet Multi-tone (DWMT)}

The structure of the analysis and synthesis filter banks of a DWMT is shown in Figure 1, where $M$ denotes the number of sub-channels. The frequency responses of the subband filters $H_{i}(\omega), i=0,1, \ldots, M-1$ are obtained by shifting in frequency domain the frequency response of the prototype filter $H(\omega)$ (with impulse response $h(n)$ ) [12]:

$h_{i}(n)=2 h(n) \cdot \cos \left((i+0.5)\left(n-\frac{N-1}{2} \frac{\pi}{M}+(-1)^{i} \frac{\pi}{4}\right)\right)$

$H_{i}(\omega)=H\left(\omega-\frac{i+0.5}{M} \pi\right) e^{j \theta}+H\left(\omega+\frac{i+0.5}{M} \pi\right) e^{-j \theta}$ $i=0,1, \ldots, M-1$,

where $\theta=-(i+0.5)\left(\frac{N-1}{2}\right) \frac{\pi}{M}+(-1)^{i} \frac{\pi}{4}$ and $N$ is the length of the prototype filter. Therefore, the prototype filter $H(\omega)$ is the only system parameter that we need to compute and optimize. There are two important considerations in the selection of the prototype filter. First, since DWMT is a MCM system with overlapping symbols in time domain, orthogonality between sub-carriers and symbols can avoid ICI and ISI and achieve perfect reconstruction (PR). Second, high frequency selectivity is preferred for the prototype filter to increase the immunity to RFI. It can be proven that PR can be ensured by using a cascade of multiple two-channel lossless lattice structures and the filter coefficient can be optimized by appropriately selecting the parameters of the lattices [13].

The commonly adopted optimization criteria are to minimize the stopband energy, minimize the maximum stopband energy or a combination of the two criteria. The corresponding objective functions of the optimization are given as:

$$
\begin{aligned}
\Phi_{1} & =\int_{(\pi / 2 M)+\delta}^{\pi}\left|H\left(e^{j \omega}\right)\right|^{2} d \omega \\
\Phi_{2} & =\max _{\omega \in[(\pi / 2 M)+\delta, \pi]}\left|H\left(e^{j \omega}\right)\right|^{2}
\end{aligned}
$$

where $\delta<\pi / 2 M$. The general design procedure using the lattices approach is as follows.

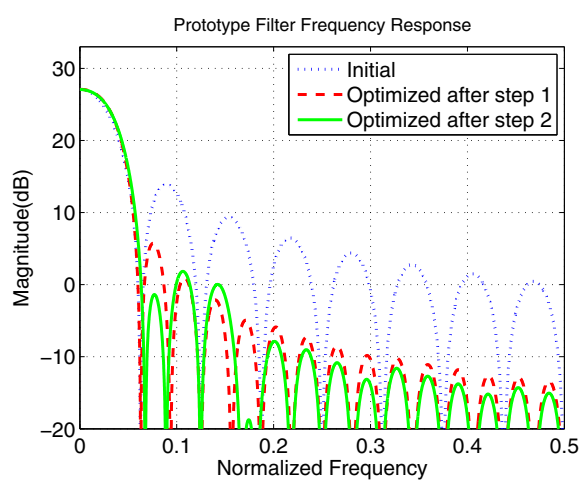

Fig. 2. An example of prototype filter frequency response with optimization

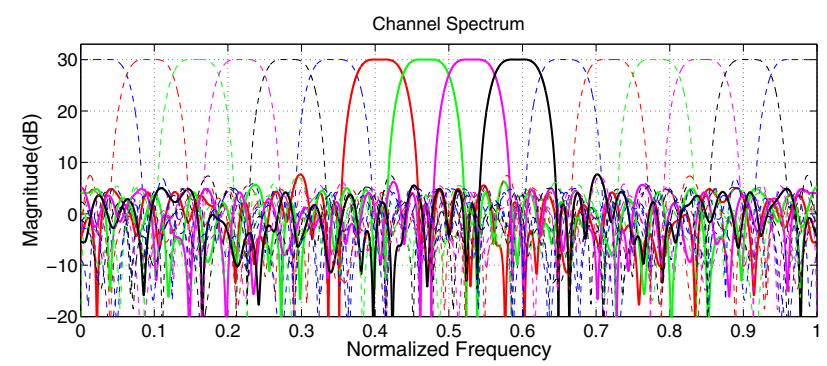

Fig. 3. Spectrum of sub-carriers of DWMT based on minimizing stopband power

- After initialization, optimize the lattice coefficients to minimize $\Phi_{1}$ to obtain the solution with minimized stopband energy.

- Based on the prototype filter derived in the last step, optimize the lattice coefficients to minimize $\Phi_{2}$ to obtain the solution with minimized maximum stopband ripple.

Figure 2 shows an example of a prototype filter response before and after the two steps of optimization. The corresponding sub-carriers spectrum of the DWMT based on the optimization method are shown in Figure 3.

\section{A Optimization SCHEME FOR DWMT}

The optimization criterion mentioned in Section II may be optimal to minimize crosstalk in DSL or interference in the applications of cognitive radio, but not necessarily in the presence of narrowband RFI. Since the RFI is relatively narrowband compared with the sub-carrier bandwidth, having low stopband energy may not be superior to having higher sidelobes with notches at the central frequency of interferer. In the case when the RFI is caused by continuously active sources, such as an AM broadcast station, it is possible to track the central frequency of the RFI disturber and adaptively optimize the DWMT [14].

Let $x_{i}$ denote the data sequence on the $i^{t h}$ sub-channel and $F_{i}\left(e^{j \omega}\right)$ denote the frequency response of the $i^{t h}$ synthesis filter, where $i=0,1, \ldots, M-1$. The frequency representation $Y$ of the transmitted signal $y$ is given by,

$$
Y\left(e^{j \omega}\right)=\sum_{i=0}^{M-1} X_{i}\left(e^{j M \omega}\right) F_{i}\left(e^{j \omega}\right)
$$


Let $H_{k}\left(e^{j \omega}\right)$ denote the frequency response of the $k^{t h}$ analysis filter. The output $V_{k}$ of the $k^{t h}$ analysis filter equals

$$
\begin{array}{r}
V_{k}\left(e^{j \omega}\right)=\left[\sum_{i=0}^{M-1} X_{i}\left(e^{j M \omega}\right) F_{i}\left(e^{j \omega}\right) H_{c}\left(e^{j \omega}\right)\right. \\
\left.+C\left(e^{j \omega}\right)+n\left(e^{j \omega}\right)\right] H_{k}\left(e^{j \omega}\right)
\end{array}
$$

where $H_{c}\left(e^{j \omega}\right)$ is the channel frequency response and $C\left(e^{j \omega}\right)$, $n\left(e^{j \omega}\right)$ are the frequency representations of the RFI and noise respectively. In (6), the interference power equals:

$$
P_{R F I}=\frac{1}{\pi} \sum_{i=0}^{M-1} \int_{0}^{\pi}\left|C\left(e^{j \omega}\right) H_{k}\left(e^{j \omega}\right)\right|^{2} d \omega
$$

Substituting from (2) for $H_{k}$, (7) can be rewritten as:

$$
\begin{array}{r}
P_{R F I}=\frac{1}{\pi} \sum_{i=0}^{M-1} \int_{0}^{\pi} \mid C\left(e^{j \omega}\right)\left[H\left(e^{j\left(\omega-\frac{i+0.5}{M} \pi\right)} e^{j \theta}\right)\right. \\
\left.+H\left(e^{j\left(\omega+\frac{i+0.5}{M} \pi\right)} e^{-j \theta}\right)\right]\left.\right|^{2} d \omega
\end{array}
$$

Since the system is designed to adapt to the time varying interference, the optimization algorithm must be computationally efficient to keep pace with the variations in the interference. To reduce the complexity of the optimization algorithm, it is assumed that the RFI is a delta function located at its central frequency and thus its bandwidth, which is approximately $1-2 \mathrm{kHz}$ due to the dominant low frequencies of the typical music signal, is ignored. We do not make this assumption in the numerical simulations where the effect of realistic RFI is studied. Therefore, (8) can be further simplified as:

$$
\begin{aligned}
P_{R F I}= & \frac{1}{\pi} \sum_{i=0}^{M-1} \int_{0}^{\pi} \mid\left[H\left(e^{j\left(\omega-\frac{i+0.5}{M} \pi\right)} e^{j \theta}\right)\right. \\
& \left.+H\left(e^{j\left(\omega+\frac{i+0.5}{M} \pi\right)} e^{-j \theta}\right)\right]\left.\delta\left(\omega-\omega_{c}\right)\right|^{2} d \omega \\
= & \frac{1}{\pi} \sum_{i=0}^{M-1} \mid H\left(e^{j\left(\omega_{c}-\frac{i+0.5}{M} \pi\right)} e^{j \theta}\right) \\
& +\left.H\left(e^{j\left(\omega_{c}+\frac{i+0.5}{M} \pi\right)} e^{-j \theta}\right)\right|^{2}
\end{aligned}
$$

where $\omega_{c}$ denotes the normalized central frequency of the RFI. It should be noted that the amplitude of the RFI is normalized to one since it is common to all sub-carriers and thus does not affect the optimization result. To minimize the power of the RFI at the receiver, (9) is the cost function of the proposed optimization criterion when the central frequency of the RFI is known. The optimization procedure with deterministic RFI can be formally stated as follows.

- Initialize the prototype filter as a rectangular window, which has stopband attenuation $A_{s} \simeq 13 \mathrm{~dB}$ and stopband edge $\omega_{s}<\pi / M$

- Select the lattice coefficients to minimize $\Phi_{\text {det }}$ the RFI power at the receiver, where $\Phi_{\text {det }}$ can be expressed as:

$$
\begin{aligned}
\Phi_{\text {det }}= & \frac{1}{\pi} \sum_{i=0}^{M-1} \mid H\left(e^{j\left(\omega_{c}-\frac{i+0.5}{M} \pi\right)} e^{j \theta}\right) \\
& +\left.H\left(e^{j\left(\omega_{c}+\frac{i+0.5}{M} \pi\right)} e^{-j \theta}\right)\right|^{2}
\end{aligned}
$$

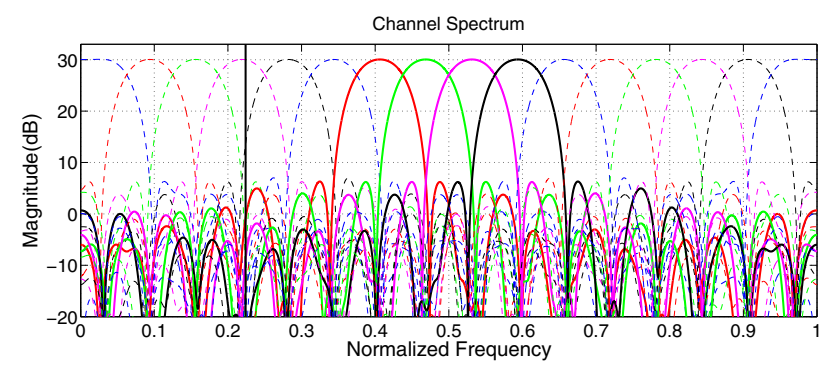

Fig. 4. Spectrum of sub-carriers of DWMT based on proposed deterministic optimization scheme. The location of the RF interference is also shown.

The central frequency, $\omega_{c}$, of the RFI is obtained by sending a training sequence and observing the noise on each subchannel at the output of the analysis filters. To reduce the computational complexity for long length prototype filters, the optimization can be divided into a two-step procedure: In the first step we can optimize a portion of the lattice sections and in the second step we can optimize for the remaining lattice sections [9]. The implementation and complexity of the optimization using the lattice filter structure is studied in [9].

Figure 4 shows an example of the sub-carrier spectrum of the DWMT using the proposed optimization. In this case, the RFI is located at a normalized frequency of 0.224 (vertical line in the plot). For a fair comparison, the filter length and the initial filter coefficients of this DWMT system are the same as the one in Section II. Unless otherwise specified, we use the same initial coefficients for all the comparisons made between DWMT systems using different optimization criteria. The DWMT system based on the proposed optimization scheme does not have higher overall frequency selectivity, since it has the same maximum sidelobe ripple, and also has more spectral overlap between sub-carriers, as compared with Figure 3. In contrast, its frequency containment is optimized to ensure that the RFI falls in the notches of most of the sub-carriers, leading to its reduced impact. Simulation results that demonstrate the superior performance of this scheme are presented in Section IV.

In some practical scenarios, the statistics of the RFI are not available. For example, if the RFI is caused by amateur radio instead of AM broadcast radio, the center frequency of the RFI can be unpredictable [14]. The reason for this unpredictability is that a ham radio operator produces a carrier only when talking and may also change the transmission frequency at any time. Besides, when the training sequence is not available for RFI estimation or there are more than one RFI sources, the optimization algorithm introduced above may be impractical. Assuming the central frequency of the RFI is unknown but the frequency band of the interference is known (which is appropriate for the scenario of amateur radio being the source of RFI), the cost function becomes the mean RFI power. Hence, the optimization procedure with probabilistic model can be formally stated as:

- Initialize the prototype filter using the same parameter as the optimization with deterministic model. 


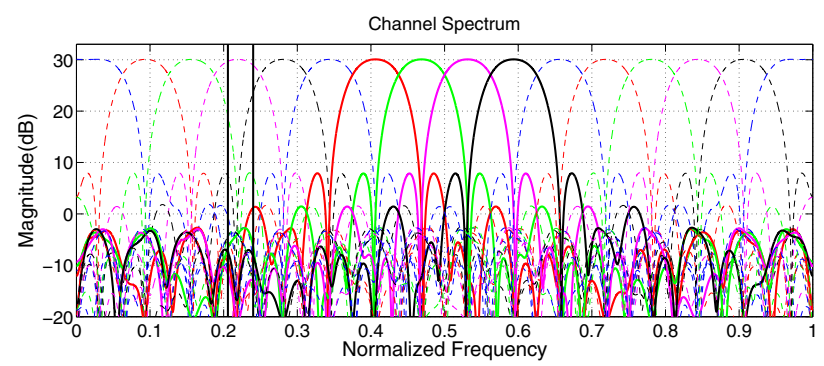

Fig. 5. Spectrum of sub-carriers of DWMT based on proposed probabilistic optimization scheme

- Optimize the lattice coefficients to minimize $\Phi_{\text {prob }}$ to obtain the solution with minimized RFI power at the receiver, where $\Phi_{\text {prob }}$ can be expressed as:

$$
\begin{array}{r}
\Phi_{\text {prob }}=\frac{1}{\pi} \frac{1}{\omega_{2}-\omega_{1}} \int_{\omega_{1}}^{\omega_{2}} \sum_{i=0}^{M-1} \mid H\left(e^{j\left(\omega-\frac{i+0.5}{M} \pi\right)} e^{j \theta}\right) \\
+\left.H\left(e^{j\left(\omega+\frac{i+0.5}{M} \pi\right)} e^{-j \theta}\right)\right|^{2} d \omega
\end{array}
$$

In this case, the normalized central frequency of the RFI is assumed to be uniformly distributed in the range $\left[\omega_{1}, \omega_{2}\right]$.

An example of sub-carrier spectrum of the DWMT system using the probabilistic cost function is shown in Figure 5. The normalized frequency range of the RFI is from 0.2057 to 0.24 , which is represented by the range between two vertical lines. This is derived from the ham band of a system with sampling frequency of $17.5 \mathrm{MHz}$ [15]. As we can see, most sub-carriers have sidelobe notches in this the frequency band, which can minimize the effect in it.

\section{Simulation Results}

A MCM system is simulated using three different modulation schemes: DMT, WPM (Coiflet-2 wavelets), and DWMT. Further, we consider three optimization approaches for DWMT - the commonly used minimization of stopband energy, and the two proposed optimization schemes. The MCM system has 128 sub-carriers and the transmitted signals have an average PSD of $-36.5 \mathrm{dBm} / \mathrm{Hz}$ and a bandwidth of $8.75 \mathrm{MHz}$. The channel is modeled as a 3000 feet, 26 gauge DSL channel and the receiver noise is modeled as AWGN with PSD of $130 \mathrm{dBm} / \mathrm{Hz}$. The RFI interferer is an amateur radio signal (50\% AM modulated carriers with a $4000 \mathrm{~Hz}$ tone) with a power level of $-25 \mathrm{dBm}$. Since the central frequency of the RFI may affect the performance of the system, simulations are performed at various RFI central frequency locations within the ham radio band.

Figure 6 shows the achievable data rate of various modulation schemes for different RFI central frequencies. The corresponding average achievable data rates are given in Table I. The data rate is expressed in percentage as the average achievable data rate with RFI over the average achievable rate without RFI. The achievable data rate is calculated based on SNR gap analysis, with the assumption of coding gain being $3.2 \mathrm{~dB}$ and margin being $6 \mathrm{~dB}$ [16]. It is clear that

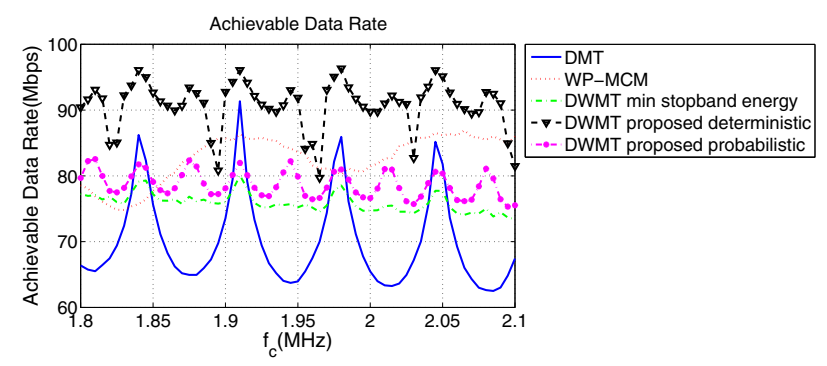

Fig. 6. Achievable data rate of various MCM schemes in the presence of RFI with different central frequencies

TABLE I

AVERAGE ACHIEVABLE DATA RATES OF VARIOUS MCM SCHEMES

\begin{tabular}{|l|l|l|l|l|l|}
\hline $\begin{array}{l}\text { MCM } \\
\text { Scheme }\end{array}$ & DMT & $\begin{array}{l}\text { WP- } \\
\text { MCM }\end{array}$ & $\begin{array}{l}\text { DWMT } \\
\text { min } \\
\text { stopband } \\
\text { energy }\end{array}$ & $\begin{array}{l}\text { DWMT } \\
\text { proposed } \\
\text { deterministic } \\
\text { model }\end{array}$ & $\begin{array}{l}\text { DWMT } \\
\text { proposed } \\
\text { probabilistic } \\
\text { model }\end{array}$ \\
\hline $\begin{array}{l}\text { Data Rate } \\
\text { (M bps) }\end{array}$ & 70 & 83 & 76 & 91 & 79 \\
\hline $\begin{array}{l}\text { Data Rate } \\
(\%)\end{array}$ & $69 \%$ & $81 \%$ & $75 \%$ & $89 \%$ & $77 \%$ \\
\hline
\end{tabular}

the DMT system without any windowing has the lowest data rate, whereas the proposed optimization approach with deterministic model achieves the highest throughput, which is about $14 \%$ higher than what is achieved with the commonly used optimization scheme. With lower complexity, by avoiding the filter coefficient adaptation and the training process, the proposed probabilistic optimization scheme still outperforms the commonly used optimization scheme. It should be noted that although the WP-MCM system has a high throughput, its incompatibility with FDD makes it impractical for DSL applications. By contrast, the proposed DWMT system not only outperforms the WP-MCM system but is also compatible with FDD operations.

The ripples in Figure 6 indicate that the center frequency of the RFI affects the performance of the MCM system. For DMT, higher data rates can be achieved when the RFI is located at the center of the main lobe of a sub-carrier, because that's also the location of notches between sidelobes of other sub-carriers. Hence, in this case it mostly affects only one subchannel. On the other hand, the system performs the worst when the RFI is located exactly in the middle between two main lobes, since both of the sub-channels would be affected, as well as the more distant subcarriers, whose sidelobes are not at their nulls at that location. However, a DWMT system with the proposed optimization approaches can achieve a higher data rate for both RFI centered at main lobe and RFI in the middle between two main lobes. The reason for this gain is that the DWMT systems have sparse sidelobes to avoid spectrum overlap with RFI. Figure 7 shows an example of the proposed DWMT system with probabilistic model, having sidelobe notches coinciding with the RFI, which is in the middle between two sub-carriers. By contrast, although the commonly adopted DWMT system may have lower overall stopband energy, it does not have sparse sidelobes to avoid narrow-band 


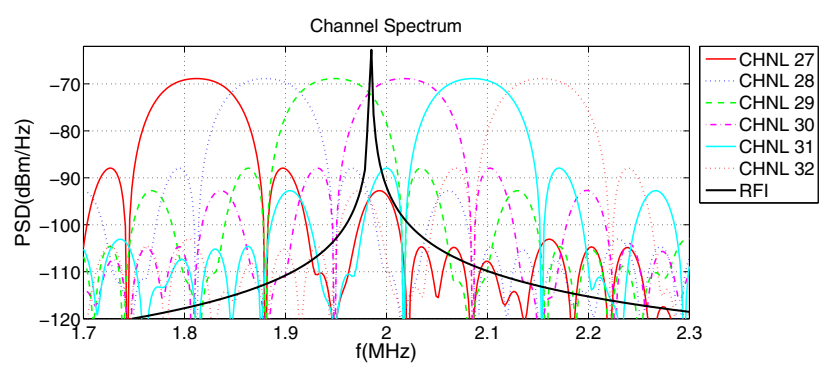

Fig. 7. Spectrum of proposed DWMT sub-carriers in the presence of RFI

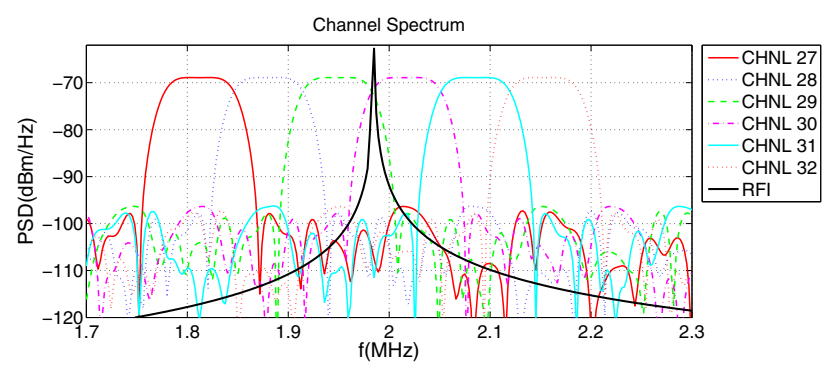

Fig. 8. Spectrum of conventional DWMT sub-carriers in the presence of RFI

interference (Figure 8). Furthermore, the optimization scheme used in the DWMT system with deterministic model exhibits a different adaptation capability to the RFI when the RFI is located at different frequencies, resulting in uneven ripples in its achievable data throughput. It is interesting to note that the WP-MCM system does not have a ripple pattern at its achievable data rate due to its unevenly distributed sub-carrier center frequency.

To further understand how the sub-carrier pulse shaping affects the vulnerability to RFI, the signal-to-interference-plusnoise ratio (SINR) of one particular case is shown in Figure 9, with RFI located at $1.96 \mathrm{MHz}$. It can be clearly seen that, in the DMT and DWMT system with conventional optimization, the RFI affects almost all the low frequency sub-carriers. By contrast, only a limited number of sub-carriers with spectral overlap with the RFI suffer from SINR degradation with the proposed optimization approaches in the DWMT system.

\section{CONCLUSion}

An optimization scheme was proposed for the design of a cosine modulated filter bank system operating in the presence of narrowband RFI. The proposed scheme is evaluated in a realistic modified DSL system, where interference of such nature is often experienced. The presented simulation results validate that, under the influence of a single known in-band RFI source, the achievable data rate, when employing the proposed scheme, is about $14 \%$ higher than what is achievable with the conventional optimization scheme. In the case when only the frequency range of the interference source is known, the proposed optimization scheme still achieves higher data rates compared to traditional approaches. Future research will consider the combined effects of RFI, ICI, ISI and crosstalk.

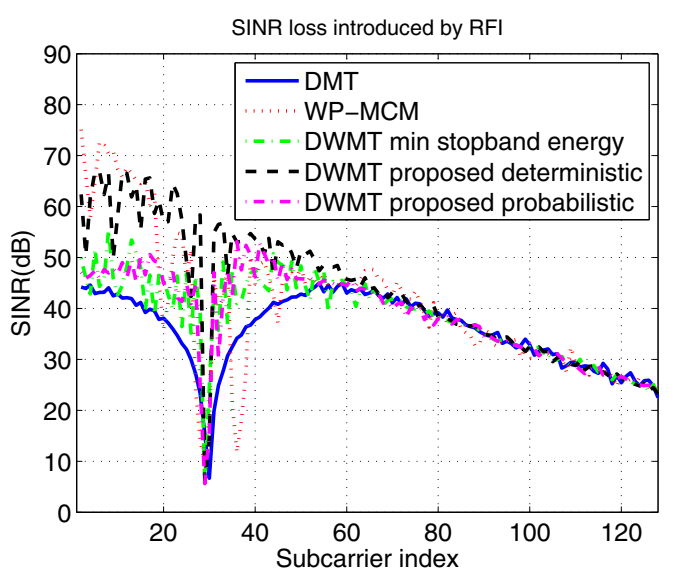

Fig. 9. SINR of various MCM schemes in the presence of RFI at $1.96 \mathrm{MHz}$

\section{ACKNOWLEDGMENT}

This research was partially funded by NSF SBIR grant IIP-0945497 and by USDA SBIR grant 2010-33610-20845.

\section{REFERENCES}

[1] I. Czajkowski, "Demographic analysis of AM broadcast RFI for a North American scenario," ANSI T1E1.4 Committee contribution number 97083, 1997.

[2] B. Farhang-Boroujeny and R. Kempter, "Multicarrier communication techniques for spectrum sensing and communication in cognitive radios," IEEE Commun. Mag., vol. 46, no. 4, pp. 80-85, Apr. 2008.

[3] A. Jamin and P. Mahönen, "Wavelet packet modulation for wireless communications," Wireless Commun. and Mobile Computing, vol. 5, no. 2, pp. 123-137, 2005.

[4] M. Gautier and J. Lienard, "Efficient Wavelet Packet Modulation for Wireless Communication," in AICT'07, Mauritius, May 2007.

[5] Y. Liang, "The application of wavelet based multi-carrier modulation in DSL systems," Master's Thesis, Southern Methodist University, 2010.

[6] B. Farhang-Boroujeny, "Multicarrier modulation with blind detection capability using cosine modulated filter banks," IEEE Trans. Commun., vol. 51, no. 12, pp. 2057-2070, Dec. 2003.

[7] A. Rizos, J. Proakis, and T. Nguyen, "Comparison of DFT and cosine modulated filter banks in multicarrier modulation," in Proc. GLOBECOM, vol. 2. San Francisco, CA: IEEE, 1994, pp. 687-691.

[8] T. Nguyen, "Digital filter bank design quadratic-constrained formulation," IEEE Trans. Signal Process., vol. 43, no. 9, pp. 2103-2108, 1995.

[9] R. Koilpillai and P. Vaidyanathan, "Cosine-modulated FIR filter banks satisfying perfect reconstruction," IEEE Trans. Signal Processing, vol. 40, no. 4, pp. 770-783, 1992.

[10] M. Furtado Jr, P. Diniz, and S. Netto, "Numerically efficient optimal design of cosine-modulated filter banks with peak-constrained leastsquares behavior," IEEE Trans. Circuits and Systems, vol. 52, no. 3, pp. 597-608, Mar. 2005.

[11] L. Lin and B. Farhang-Boroujeny, "Cosine-Modulated Multitone for Very-High-Speed Digital Subscriber Lines," EURASIP Journal on Applied Signal Process., vol. 2006, pp. 1-17, 2006.

[12] N. J. Fliege, Multirate Digital Signal Processing. Wiley, 2000.

[13] P. Vaidyanathan, "Passive cascaded-lattice structures for low-sensitivity FIR filter design, with applications to filter banks," IEEE Trans. Circuits and Systems, vol. 33, no. 11, pp. 1045-1064, Nov. 1986.

[14] P. Golden, H. Dedieu, and K. S. Jacobsen, Fundamentals of DSL Technology. Auerbach Publications, 2004.

[15] K. T. Foster and J. W. Cook, "The Radio Frequency Interference (RFI) environment for very high-rate transmission over metallic access wirepairs," ANSI T1E1.4 Committee contribution number 95020, 1995.

[16] J. M. Cioffi, "A multicarrier primer," ANSI TIE1.4 Committee Contribution number 91-157, 1991. 